\title{
Políticas universitarias y neoliberalismo. Resultados y tendencias en la universidad latinoamericana*
}

DOI: https://doi.org/10.32870/dse.v0i9.294

\section{Liberio Victorino Ramírez** Rocío Ángeles Atriano Mendieta*** Héctor Rueda Hernández****}

RESUMEN: Se parte de la premisa que las universidades en América Latina crecieron a nivel pregrado en los años setenta y ochenta, pero en su nivel de posgrado se expandieron más en los noventa del siglo XX y los primeros catorce años del siglo XXI al calor de la constitución de los diversos bloques regionales en el continente. Y esta misma asociación ha acompañado al respaldo de las universidades en su crecimiento, en sus políticas de evaluación, de investigación, de acreditación, entre otras. Estos bloques se han desenvuelto en las siguientes orientaciones: el Mercosur liderado por Brasil y Argentina, incorporando otros países del cono sur y con fuertes nexos vía tratados comerciales con países de distintas regiones del mundo; el grupo Alternativa Bolivariana de las Américas (ALBA), liderado por Venezuela, agrupando a ciertos países como Ecuador, Nicaragua, Honduras, entre otros; y el tercero sin un verdadero liderazgo a nivel latinoamericano, aunque buscando y promoviendo el liderazgo de Estados Unidos de Norteamérica que incluye países como Colombia, Costa Rica, Panamá y México. El impacto de las políticas universitarias estará influido por estas orientaciones. El objetivo principal de este trabajo es hacer un balance de las políticas públicas universitarias de los 9o, describir sus resultados y argumentar los escenarios posibles y deseables hacia el 2030 con la finalidad de analizar las nuevas fuerzas del cambio en complemento a las llamadas "reformas de segunda generación" para mejorar la calidad de las instituciones universitarias de pregrado y posgrado en la región latinoamericana. Palabras clave: políticas universitarias, neoliberalismo, cambios, tendencias y escenarios.

ABSTRACT: It starts from the premise that the universities in Latin America grew at undergraduate level in the seventies and eighties, but in its postgraduate level expanded more in the nineties of the twentieth century and the first fifteen years of the twenty first century through the development of the constitution of the diverse regional blocks in the continent. And this same association has accompanied to the endorsement of the universities in their growth, in their assessment policies, of research, accreditation, among others. These blocks have developed in the following directions: The Mercosur led by Brazil and Argentina, incorporating other countries in the South cone of America and with strong links via trade agreements with countries from different regions of the world; the Bolivarian Alternative for the Americas (ALBA), led by Venezuela, grouping to certain countries such as Ecuador, Nicaragua, Honduras, among others; and the third without a real leadership in Latin American, although seeking

* Con algunos cambios este artículo se basa en la ponencia presentada en el workshop internacional "Balance de las políticas sociales en Iberoamérica" de la Red Iberoamericana de Estudios de Políticas Sociales, a realizarse del 2 al 5 de abril de 2014 en la Universidad de Colima, Colima, México.

** Profesor-investigador, Universidad Autónoma Chapingo, investigador nacional por el Sistema Nacional de Investigadores (SIN) del CONACYT-SEP de México. Email: victorinoramrezliberio@yahoo.com.mx Subdirección de investigación, UACh, tel. 01595 9521500 ext. 5707. Cel. 5554535328.

*** Docente y coordinadora de Posgrado e Investigación de la Universidad Pedagógica Nacional, sede Atizapán, México. Email: extension41@hotmail.com.

${ }^{* * * *}$ http://forum.wordreference.com/showthread.php?t=1456637\&langid=24. 
and promoting the leadership of the United States of North America including countries such as Colombia, Costa Rica, Panama and Mexico. The impact of the university policies will be influenced by these guidelines. The main objective of this work is to take stock of the university public policies of the nineties, describe their results and argue the possible and desirable scenarios by 2030 in order to analyze the new forces of change in addition to the so-called "second generation reforms" to improve the quality of the university undergraduate and postgraduate institutions in the Latin American region. Key words: university policies, neoliberalism, changes, tendencies and scenes.

\section{Introducción}

Aunque se precisa la posición teórica y metodológica que adopta este estudio, es muy importante retomar la necesaria utilidad para analizar estos conflictos desde una perspectiva comparada, "lo cual implica reconocer y conocer los procesos y actores que dan sentido a estructuras académicas formalmente similares. Sin embargo, los actores y sus orientaciones así como los valores y las prácticas que explican el devenir universitario son notablemente diferentes, aunque curiosamente algunos indicadores de resultados sean similares para el conjunto de la región" (Krotsch, 2006). En consecuencia podríamos preguntarnos si ¿existe un patrón socioeconómico y cultural común a la región que determine un modelo de universidad? La respuesta tentativa sería que sí, guardando sus distancias, sobre todo en el nivel procesual práctico de las políticas universitarias. Pero en lo que respecta a los rasgos regionales y en los seis ejes analíticos que se mencionan, además de las políticas de rasgos neoliberales que se están aplicando, difícilmente escapan las universidades, pero sobre todo a los programas de posgrados; por ello vale la pena mencionarlos como estándares globales.

En una visión de la experiencia mexicana tomado como eje central de análisis, así como la complementariedad y comparación con universidades públicas de similar desarrollo de los países en cuestión, se hace por la vía documental una indagación de las experiencias de estas universidades en una lógica de observación de tendencias más recientes como procesos de aceptación, rechazo y resistencias de estas políticas educativas en transición.

En la realidad universitaria, aunque hay más políticas universitarias como el financiamiento, la cobertura, entre otras, las primeras seis políticas públicas educativas conforman los principales focos rojos en la agenda universitaria. El conjunto de reflexiones sobre ellas dan cuenta del comportamiento de estas universidades en transición de la región latinoamericana, el cual se mueve de un Estado de bienestar a uno de rasgos neoliberales y en otras naciones transitan de uno neoliberal a otro de rasgos socialdemocrátas en los últimos 20 años de vida universitaria; por tanto se busca documentar ¿cuáles son los resultados de las principales políticas públicas educativas que siguen influyendo en la transformación de las instituciones de educación superior y por ende, en el posgrado? Y ¿cómo las universidades aceptan, rechazan o se resisten ante la orientación estatal en los singulares países del continente latinoamericano?

Para una mejor exposición se enumeran los alcances, las apreciaciones críticas, así como las opciones para su mejora de las ocho políticas educativas públicas hacia las universidades, así como la necesaria delimitación teórica. En orden de aparición se tiene los siguientes apartados: Pri- 
vatización, financiamiento y crecimiento; Políticas de evaluación y acreditación; Vinculación interinstitucional, Cooperación internacional; Investigación sobre las IES; Internacionalización del posgrado; Incorporación de la educación mediadas por las TIC; así como incorporamos algunos escenarios posibles y deseables para el desarrollo de los estudios de posgrado en el continente en función de los ejes analíticos referidos, así como en la prospectiva recurrente y, por último, las Reflexiones finales.

\section{Principales plíticas universitarias Privatización, financiamiento y crecimiento}

En el contexto de políticas de crecimiento del mercado y achicamientos de la sociedad civil y del Estado los sistemas educativos del nivel superior devinieron en un giro hacia el fortalecimiento de la matrícula en las instituciones de educación superior (IES), e intermitentemente los contrasentidos del financiamiento precarios a las IES públicas y el apoyo incluso financiero a las privadas se registraron los siguientes cambios en nuestro continente:

a) En los años sesenta del siglo XX, Brasil, Colombia y Chile contaban con menos del $20 \%$ de la matrícula en el sector privado mientras que en los noventa la mayoría de los países alcanzaba ese porcentaje, en tanto que esos tres primeros países lograban ya un poco más de $50 \%$.

Pese a ello el sector del posgrado público creció y se despegó con una impresionante cantidad de 9 mil programas de posgrado de Especialidad, Maestrías y Doctorados. La gran concentración sin lugar a dudas se daba en el nivel de la Maestría con 51\%, mientras que en la Licenciatura en México o pregrado para el resto de los países de la región latinoamericana no creció tanto como en el posgrado. En este cuarto nivel educativo la concentración de la matrícula se dio en el sector público con $75 \%$ de su concentración. Sin embargo, países como Brasil y México van a la cabeza con $71 \%$ de la matrícula total en Maestría y Doctorado.

b) El promedio de crecimiento en América Latina alcanzó 17.4\% parecido al promedio mundial pero muy por abajo del de los países desarrollados que llegan a 60\% de la cobertura (UNESCO, 1998). Si bien países como Argentina mantienen el modelo universal de 35\%, hay otros países de la región como Bolivia, Colombia, Costa Rica, Chile, Ecuador, El Salvador, República Dominicana, Uruguay y Venezuela que se mueven más en el modelo de masas que fluctúa entre 15 y 35\% de su capacidad. Peor aún en los noventa todavía había países que mantenían cobertura por debajo de 15\%, que se pueden ubicar en el modelo de universidad élite, como Brasil, Paraguay, México, Nicaragua, Honduras y Guatemala.

c) El crecimiento de las universidades también se dio en modalidades diferentes a las convencionales. Distintas universidades de las subregiones se organizaron para implementar y fortalecer la educación superior 
Liberio Victorino Ramírez, Rocío Ángeles Atriano Mendieta y Héctor Rueda Hernández

mediadas por las Tecnologías de la Información y Comunicación (TIC). Organizadas en redes promovieron interesantes programas que animaron el crecimiento del sector, mediante la modalidad de educación a distancia o virtual. Así celebraron convenios de cooperación en la materia entre IES latinoamericanas y universidades de la Unión Europea (UE) en materia de universidad virtual, mediante la conformación de la Red UNIVERCO, coordinada por universidades argentinas con participación de Brasil, Gran Bretaña e Israel.

Una de las redes más conocidas sin duda fue la Red de Información Iberoamericana (RIBIE) con participación de Argentina, Brasil, Colombia, Venezuela, México (Silvio, 2003).

\section{Políticas de evaluación y acreditación}

Para diversos estudiosos de la materia, la década de los noventa ha sido catalogada como la etapa de la acentuación de las políticas de la evaluación y acreditación de los sistemas educativos universitarios (Kent, 1998; Victorino, 2006). Estas acciones a fin de cuentas como medidas de control y organización de los diversos sistemas del nivel superior o de pregrado fue desarrollado ampliamente en Argentina, Brasil, Colombia, Costa Rica, Chile y México. Con una clara prioridad que respondió a cuestiones internas de índole política para racionalizar los presupuestos de financiamiento. En ese sendero unos países avanzaron más en las evaluaciones diagnósticas, en tanto otros le dieron gran prioridad a las de evaluación, para las acreditaciones nacionales, como Chile y Colombia.

Hay diversas experiencias sobre las políticas sociales de evaluación como parte del proceso para la acreditación de programas académicos en la región que, independientemente de los tipos de gobiernos nacionales de las universidades fueron impactantes estas medidas, que en muchas universidades latinoamericanas se convirtieron más tarde en políticas para el aseguramiento de la calidad académica (Victorino, 2006).

En el caso específico de los estudios de posgrado en la región, ha tenido distintos ritmos de impulsos que transitan de lo meramente interinstitucional a lo estrictamente de cooperación e intercambio, con universidades que comparten un mismo campo de estudio y áreas de conocimiento con miras a compartir, incluso, programas de posgrados conjuntos entre ellas.

\section{Cooperación internacional}

Las acciones de intercambio entre las universidades, han venido a darle nuevos aires a lo que se conoce cada vez en la mirada global como la vinculación internacional por diferentes vías, tanto meramente institucionales como las acciones animadas por las redes sociales académicas, y en algunos casos temáticas, que brotan de los acuerdos y pactos de las propias comunidades académicas y científicas en el contexto de las universidades.

En los noventa algo común y particular en las IES de la región fue la cooperación internacional de estas con sus IES similares de países de la Unión Europea (UE). Al calor de los nuevos 
impulsos por aplicar acciones de movilidad de estudiantes y docentes investigadores, varias IES de la región latinoamericana aprovecharon los apoyos de programas fuertes de la UE. Sin duda son reconocidos los impactos de programas como el América Latina Formación Académica (ALFA) conocido en el 2004. Este programa financiado por la UE promueve la cooperación e integración de las IES europeas con las propias de América Latina. La primera parte estaba organizada desde 1995 en tanto que la segunda debía ser evaluable entre los años de 2000 y 2005. Su focalización era la movilidad en los estudios de posgrados para cursar programas hacia la Especialización, Maestría y Doctorado. Pese a que es difícil conocer el resultado de su impacto, sabemos que todavía en el 2007 se apoyaron algunos programas de estudios doctorales en su modalidad de educación no convencional, especialmente a distancia o virtual (DOCINADE, 2008).

Otro programa importante, aunque de menor cobertura y difusión, fue el plan COLUMBOS que también en la década del noventa fue firmado por 130 rectores de diversas IES y por 32 países de ambas regiones del mundo. Aunque se desconoce sus resultados en cantidad de programas y recursos económicos, en varias IES de la región todavía se comentan entre profesores las ventajas de quienes solicitan apoyos diversos para estudios de posgrado en el contexto educativo de este plan.

\section{Investigación sobre las IES}

Desde los noventa comenzaron a tomar cierta importancia la organización de la investigación sobre las propias IES como objeto de estudio mediante una nueva forma de organización ahora basadas en redes sociales. Una de las primeras redes que se conoce en el continente con estos rasgos es la Red de Investigación sobre Estudios de la Universidad (RISEU) promovida por investigadores mexicanos y venezolanos, cuyos proyectos de investigación dan prioridad a los estudios comparados de las IES de la región latinoamericana. De manera complementaria estimulan esfuerzos para incorporar de nueva cuenta la investigación inter y transdisciplinaria (Follari, 1999). Dichos estudios se mueven en temas relevantes sobre Gestión, Organización y Evaluación, en países como Argentina, Brasil, Venezuela y México.

En la década del noventa, a diferencia del planteamiento de Ezcurra (2008) quien sostiene que no hay una agenda de la investigación de los universitarios para las IES de América Latina, el grupo de la RISEU ha publicado en varias ocasiones no sólo la necesidad de dicha agenda sino la propuesta de un plan para el grueso de las IES de la región latinoamericana con énfasis en Argentina, Brasil y México (Kent, 1997).

En este contexto de indagación sobre la problemática de le educación superior no se puede olvidar vastos libros financiados y publicados por la Fundación Ford, bajo la coordinación de José Joaquín Brunner y Jorge Balám, sobre políticas comparadas en cinco países de la región como México, Chile, Brasil, Argentina y Colombia (Kent, 1997). Otros donde se comparan, aunque sea mediante un enfoque cuantitativo, a 19 países de la región auspiciados por la Cátedra UNESCO de estudiosos de la Universidad Central de Venezuela (García, 1998). 
En esta década para el fortalecimiento de los estudios de las universidades como organizaciones de una gran diversidad, se reconoce la influencia de planteamientos como del norteamericano Burton Klark (1998) sobre la organización y problemas de vinculación de la docencia y la investigación en las universidades del continente latinoamericano. Una de las conclusiones de este sociólogo e investigador en el campo de la educación sostiene que la organización de algunas IES resultan atípicas comparadas con las universidades estadunidenses, no tanto por mantener algunas de ellas, como el caso mexicano vinculada orgánicamente al nivel de educación media superior con el nivel superior, sino por sostener con gran preocupación otras funciones sustantivas como el servicio universitarios y la difusión de la cultura. En fin, Clark (quien desgraciadamente fallece en los primeros días de noviembre de 2009, para quien desde este ensayo le rendimos un homenaje como profesor Emérito de la Universidad de California, USA) señala que las universidades, en algunos casos son IES de una "anarquía organizada" que se mueven aun con raquíticos presupuestos de los gobiernos federal y estatales, pero mantienen una heterogeneidad de actividades en sus funciones sustantivas y sociales.

No podría faltar la influencia en esta propia década del siglo XX de un discurso sobre el enfoque de la modernidad/posmodernidad, manejado en países como Francia, de teóricos como Lyotard y Derrida, desde luego en América Latina son más conocidos en esta percepción por los intelectuales de la llamada Teoría Crítica, especialmente representado por Jurgen Habermas. Al mismo tiempo que se fueron conociendo los planteamientos de otro de los pensadores franceses más fresco y un poco alejado del marxismo y posmarxismo como Edgar Morín, en sus planteamientos más elementales de la teoría de la complejidad o más conocido como el pensamiento complejo (Morín, 2004), aplicada al estudio de las organizaciones universitarias. Debemos reconocer como propuestas emergentes a un enfoque diferente a los demás, que centra su pensamiento sobre el llamado enfoque de épocas o visiones de mundo, que compara las visiones de pensamiento único frente a las nuevas realidades que se están viviendo en una época emergente conocida como la Era de la información (Castells, 2000) o el informacionalismo (De Souza, 2004) y que ha tenido impacto en algunos estudios de las instituciones de educación agropecuarias del nivel superior (IEAS) de la región. Dicha época emergente se gesta desde los años sesenta y seguramente llevará su desarrollo muchos años en el futuro del siglo XXI. Su principal indicador son los avances científicos tecnológicos, esencialmente las TIC y su fortalecimiento en el campo de la docencia y la investigación, son precisamente el multiuso de la red de Internet y con ello las redes sociales en la investigación y la innovación científica en diversos campos de estudios en la Universidad latinoamericana (De Souza, 2004).

\section{Internacionalización del posgrado}

En este aspecto hacemos referencia al nivel latinoamericano, porque la Universidad Autónoma Chapingo (UACh) de México, es comparable con algunas IES de países de similar desarrollo que 
México, tomando las instituciones más representativas en cuanto a la vinculación de la investigación y el posgrado, en los niveles de licenciatura y los estudios del cuarto nivel considerado el posgrado, en la idea de que esta dupla es inseparable en el desarrollo de las instituciones de educación superior (IEAS) en cualquier parte del mundo. Desde luego se especifica al posgrado (Maestría y Doctorado) porque es considerado como el principal espacio formal para la formación de investigadores.

Según José Joaquín Brunner (2007), en "Universidad y Sociedad en América Latina”, al centrar su análisis sobre las grandes y complejas universidades que han visto incrementar su matrícula en los estudios de posgrado (Maestría y Doctorado), argumenta que se pueden distinguir en la realidad latinoamericana, tres tipos de universidades:

$1^{\circ}$. Universidades complejas, comparables a las de los países desarrollados, con un buen equilibrio entre docencia (posgrado), investigación y servicio a la sociedad. Se sitúan casi exclusivamente en vinculación a cuatro grandes clusters: México, DF, (y su zona metropolitana); Santiago de Chile, Buenos Aires, Argentina; y unas cuantas ciudades de Brasil como Sao Paulo, Río de Janeiro, Campinas, entre otras. Con todas ellas no llegan a $3 \%$.

$2^{\circ}$. Universidades importantes en las principales ciudades de todo el continente, bien organizadas, con selectos cuadros de profesores e investigadores de tiempo completo, en su gran mayoría con grados de maestro en Ciencias y doctores, y un impacto significativo en sus respectivos países, pero más centradas en docencia del posgrado y que sólo tienen algunas unidades de investigación aisladas. Aunque muchas de ellas con cierto desarrollo de investigación y formación de investigadores de vinculación internacional. Estimamos que constituyen no más de 7\% de la realidad universitaria latinoamericana.

$3^{\circ}$. El 90\% restante son universidades centradas en la docencia en licenciatura, o pregrado, con poquísimo desarrollo del posgrado-investigación y un aporte a los contextos sociales muy limitado.

En México, la UNAM, la UAM, el IPN y la UACh, entran en la categoría de universidades complejas que han vinculado los estudios de posgrado a la investigación y el servicio. Lo curioso es que si bien están colocadas en el peldaño de universidades complejas vinculadas al posgrado y a la investigación, el empeño sigue siendo lograr un mayor impacto internacional, entablar convenios con universidades de otros países para ofrecer proyectos de investigación colectivos, e impulsar y desarrollar programas de formación de investigadores de manera compartida, con créditos de doble o triple reconocimientos y por tanto, grados homologados con validez oficial internacionalmente.

En este contexto en México, la UACh como la principal universidad de orientación agrícola, viene impulsando políticas educativas que la colocan tanto en la licenciatura como en el posgrado como una institución con reconocimiento formal por la acreditación de sus programas académicos, el reconocimiento de sus programas de posgrado como programas de calidad. Dicho reconocimiento goza ya de pertenencia en todos sus programas de posgrado orientados a la investigación en el Pa- 
drón Nacional de Posgrado de Calidad (Conacyt, 2007). En la licenciatura con el reconocimiento por la acreditación nacional de casi la totalidad de sus programas académicos acreditados a fin de cuentas por la Secretaría de Educación Pública de México. Estas acciones son el primer paso para buscar la internacionalización de sus carreras y programas académicos.

La internacionalización del posgrado en la globalización significa incorporación de rasgos académicos con estándares de alta calidad que el mundo científico-tecnológico y económico-social demanda. La internacionalización educativa busca una formación común de los futuros profesionales e investigadores para que en el corto plazo se acceda, sin conflictos para el intercambio y movilidad magisterial y estudiantil, a los distintos países incluidos en el TLCAN y aún a países de bloques regionales como la Unión Europea, la Cuenca del Pacífico o América Latina (Victorino, 2008).

En cuanto al diagnóstico nacional, regional e institucional se parte de las siguientes reflexiones: el éxito de las potencias económicas se debe, en gran medida, a su gran desarrollo científico-tecnológico y a la fortaleza de sus universidades. Pero hasta en esos países, la asignación de presupuestos gubernamentales para dichas actividades sigue siendo un problema que reclama de continuas negociaciones entre el aparato gubernamental y las pretensiones de los investigadores y de las propias instituciones.

En México, el presupuesto para la ciencia es raquítico y no parece que, ante las crisis económicas recurrentes, pueda mejorar en el futuro cercano. Las recomendaciones internacionales señalan que se requiere destinar al menos $1 \%$ del Producto Interno Bruto (PIB) para el despegue científico de un país menos desarrollado; en el nuestro, apenas se otorgó 0.33 por ciento en el sexenio foxista 2000-2006. En el actual gobierno, 2006-2012, en el primer semestre de 2008 en plena crisis alimentaria mundial, parece indicar que las políticas públicas hacia el posgrado se castigarán una vez más, por tratar de mitigar las grandes prioridades de alimentación y subsidios a la gasolina, dísel, entre otros productos que benefician también a la clase media y sectores acomodados (Victorino, 2009).

Son factores adversos para la enseñanza en nuestro país la exigüidad de condiciones socioeconómicas que afectan primordialmente a estudiantes pobres que truncan sus estudios (alrededor de 10 millones de mexicanos sobreviven con un dólar diario). Al igual que en otros países, la motivación económica para los profesores es menor en muchas instituciones docentes que la proporcionada por la industria, y este hecho origina rotación desfavorable del personal. Lo que atenúa la gran vocación y conciencia social de los profesores hacia la trascendente labor formativa de los profesionales e investigadores en México.

En cuanto a la economía nacional, la aplicabilidad de las normas económicas en las distintas partes del mundo ha marginado a los sectores sociales mayoritarios, sectores medios y pobres del mundo. Desde que se instauró el neoliberalismo en los distintos bloques regionales, se han registrado varias crisis financieras y no se han podido superar problemas de carteras vencidas, endeuda- 
miento de los sectores productivos, y tampoco se ha reactivado la inversión hacia la producción. En México las distintas promesas de beneficio para los sectores populares, apoyo a la educación pública, inversión al campo, programas específicos como PROCAMPO, y con una transformación estatal mediante cambios a los artículos constitucionales como el $3^{\circ}$, referente a la educación pública, gratuita y laica; el artículo $27^{\circ}$, relacionado con el cambio en la tenencia de la tierra ejidal y ahora, una provocación a desaparecer el ejido, entre otros, indican que difícilmente lograrán sus objetivos; mientras tanto, se ha agudizado en el sentimiento y proyectos sociales y culturales del sector rural, $24 \%$ de la población según INEGI (2007), con quienes la UACh tiene un gran compromiso social.

Pese a lo alcanzado en materia de evaluación para la acreditación nacional en la licenciatura y a su pertenencia al Padrón Nacional de Posgrado de Calidad del Conacyt, la calidad en la UACh, especialmente en el posgrado no puede resolverse sólo con mecanismos rígidos de evaluación. La calidad comprende un conocimiento de muchos cambios que va desde la filosofía misma, con visión, hasta una planeación estratégica. El posgrado en la UACh, sobre todo el orientado a la investigación, ha logrado su incorporación al Padrón Nacional de Posgrado de Calidad (PNPC) del Conacyt, esencial para mejorar la calidad y lograr niveles de excelencia. Es un reconocimiento de que la educación que se imparte en la UACh se base en una inversión que posibilite un constante mejoramiento del desarrollo de las funciones sustantivas y adjetivas con el propósito de alcanzar mayor eficiencia en la optimación de recursos humanos y financieros; así como eficacia en el cumplimiento de objetivos y metas en los procesos educativos y de su impacto social.

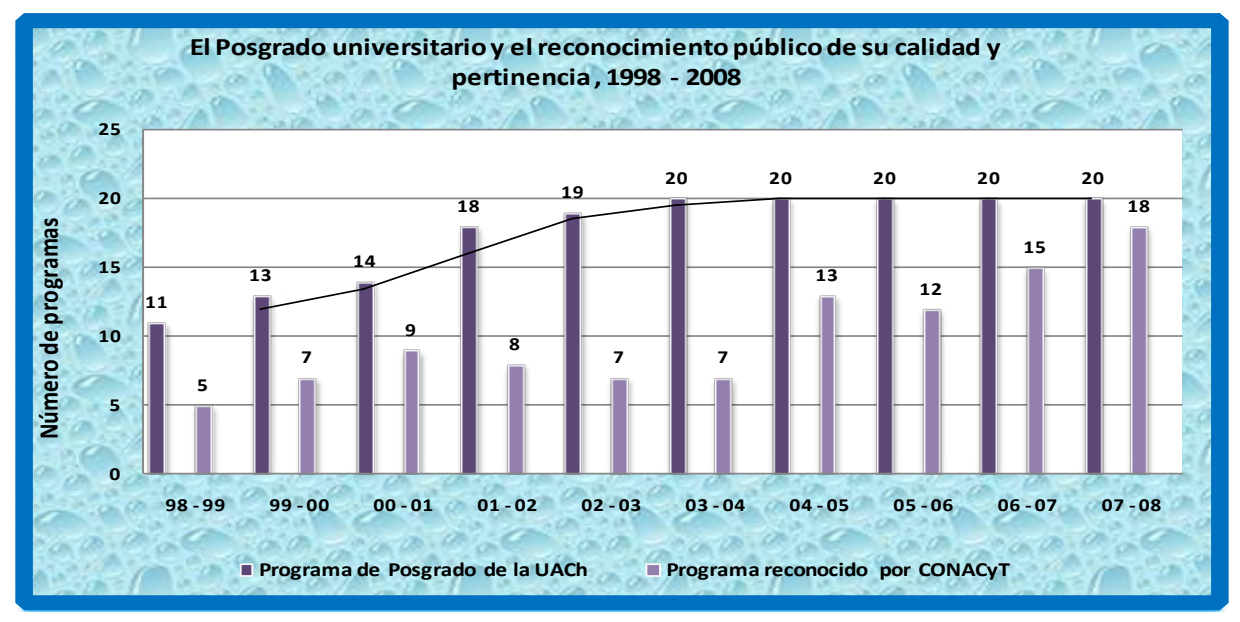

Fuente: UPOM-UACh. 1998-2008. Anuario Estadístico (PDI, 2009-2025).

Un hecho muy importante es que durante el periodo 2003-2006, en lo referente a sus programas de posgrado, la Universidad Autónoma Chapingo de México fue una de las mejores evaluadas en- 
tre las universidades públicas del país, especialmente en el área agropecuaria. Con estos frutos la UACh afianza su reconocimiento público y su pertinencia social; aun así, es indispensable que los integrantes de la universidad participen en los órganos de evaluación y contribuyan en la formulación de criterios de calidad que correspondan en mayor medida con los objetivos institucionales y respondan adecuadamente a las problemáticas de los procesos productivos, económicos, ambientales, sociales, culturales y del medio rural (PDI, 2009-2025).

En el año 2007, los 18 (100\%) programas de posgrado con orientación hacia la investigación fueron considerados como de alto rendimiento y de excelencia por el Conacyt están incluidos en su PNPC. Desgraciadamente las Maestrías en Procesos Educativos y la Maestría en Agroempresas sólo son de orientación profesionalizantes o de especialización, por lo que no pueden aspirar a estar dentro del PNP del Conacyt en las mismas condiciones que los de orientación hacia la investigación (PDI, 2009-2025). En concordancia con las políticas establecidas por el Conacyt y la SEP, la mayoría de los programas responden de manera creciente a los requerimientos del mercado y del sector productivo más dinámico del agro mexicano, pero a la vez, se margina a otros que pudieran ser compatibles con los fines institucionales.

Como consecuencia, se concibe una ambigua conformación disciplinaria del posgrado al no ser claros los contenidos curriculares de algunas Maestrías y Doctorados que tienen un mismo objeto de estudio, ni los niveles de confluencia de otros programas, como Desarrollo Rural Regional, Economía del Desarrollo Rural y Sociología Rural (PDI, 2009-2025).

\section{Financiamiento, producción y generación de conocimientos en las universidades de referencia}

En función de los argumentos anteriores de acuerdo a Krotsch (2006) se quiere sólo subrayar un tema muy complejo que tiene que ver con la producción de conocimiento, con la ciencia y la cultura regional en la Argentina y en América Latina. Krotsch resalta el hecho de que la universidad y la sociedad, o más bien el vínculo entre universidad, sociedad y economía que hemos conformado históricamente, ha sido poco proclive al desarrollo científico y tecnológico. Distintos autores como Risieri, Frondzi señalaba en 1956 que aún teníamos una universidad colonial. Steger aludía también refiriéndose al modelo de universidad latinoamericana como el "modelo de la universidad de los abogados". Hoy este modelo trasmuta en formas diferentes pero no ha cambiado en lo esencial lo que también es válido para el conjunto de América Latina, cuyos sistemas se han masificado y complejizado sin duda en términos de relación público-privado, multiplicación de instituciones, creación de organismos de aseguramiento de la calidad y las constantes apelaciones a este último término en los debates regionales. Para terminar, algunos señalamientos respecto a la pérdida de protagonismo de la universidad latinoamericana y de los desafíos que debemos asumir frente a los retos del futuro y la pérdida de protagonismo de la universidad latinoamericana. A pesar de que los números se han multiplicado de manera espectacular, tanto la matrícula como las instituciones 
públicas y privadas, las reformas neoliberales de la última década tampoco han modificado (bis a bis) el perfil de nuestra universidad.

Veamos algunos datos vinculados al papel del desarrollo científico tecnológico en la región y el protagonismo de nuestros países, que ha disminuido a nivel global fundamentalmente por la emergencia del Este Asiático en el panorama de la producción de conocimiento.

1) En materia de gasto sobre el producto interno bruto (PIB) para el año 2001 si consideramos entre los países desarrollados USA, Alemania, Japón, Corea y entre los periféricos a Argentina, México y Chile las diferencias no son demasiado significativas, pues varían entre 7\% en USA y Chile, 6\% para Argentina y 4.7\% para Japón, todos fundamentalmente como aporte público. Aunque en México el PIB dedicado a Ciencia y Tecnología (CyT) no llega ni a 1\% (Victorino, 2008).

2) En materia de gasto en investigación y desarrollo (1\&D, 2002) la diferencia es muy significativa a favor de los países desarrollados. En primer lugar Japón con 3\%, Corea con prácticamente el mismo porcentaje y Alemania y USA con 2.40 del PIB; Argentina, Brasil, México y Chile están muy por debajo de estos porcentajes. Todos por debajo de 1\% salvo Brasil. Argentina en este caso está por debajo de los países latinoamericanos mencionados. Esto se está modificando pero la situación es básicamente similar. Lo significativo aquí es que en los países desarrollados más de 70\% es aportado por las empresas en América Latina, el financiamiento es exactamente inverso. En la ejecución del gasto o la inversión en ciencia pasa algo similar. 3) En relación a los investigadores en $\mathrm{C}$ y T, considerados como \% por 1.000 de la PEA las diferencias son también muy significativas, Japón tiene $8 \%$ de la PEA y Argentina que tiene el mayor \% de los países latinoamericanos que hemos considerado no llega a 2\%, siendo mucho menor en el resto de los países latinoamericanos. Si analizamos los datos por sector veremos que los investigadores se localizan en el sector privado predominantemente en los países desarrollados, y en la universidad y gobierno en los países centrales. 4) En términos de artículos publicados en revistas científicas, indexados por el Institute for Scientific Information (ISI) 1981-2002, podemos observar ante todo el crecimiento espectacular de Corea. Es impactante la distancia en números absolutos de la producción en América Latina respecto de los países centrales. Un país pequeño cono Corea produce en torno de los 15,000 artículos en 2002; Argentina 468, de manera similar a México y menos que Brasil. Aunque no está en el cuadro si consideramos la productividad por investigador, la Argentina tiene un desempeño destacado, aunque un crecimiento porcentual menor que el de México y Brasil.

5) En términos de crecimiento, considerando 1997 de artículos publicados, el ranking es ocupado por China y en segundo lugar Corea y luego Japón. Brasil está en el $7^{\circ}$ lugar y México en el 18. Es importante el crecimiento de España, Turquía y por supuesto la India.

Algo muy importante, respecto de lo que no se dispone suficiente información, es la ocupación de los ingenieros al egreso. Es un dato muy significativo para comprender, aún más que la tasa de graduados por área disciplinaria, para comprender el desarrollo de la investigación y el desarrollo. 
Tal y como se ha argumentado brevemente el papel de América Latina comparado con países desarrollados de Europa y Norteamérica es precario y ha perdido terreno desde el punto de vista de su producción científica, que no se puede explicar, evidentemente ,desde el propio campo científico, sino que tiene que ser analizado en el marco de los patrones de desarrollo, pero también, cosa que no se hace en relación a los patrones culturales de América Latina.

En síntesis, se puede decir que la Universidad latinoamericana está suspendida en una triple crisis: de su hegemonía, de su legitimidad y también de la institución. Esta crisis tal como se la ha caracterizado se refiere fundamentalmente a la Universidad tradicional. En la tradicional universidad nacional para el caso de Argentina cuyo paradigma son las de Córdoba, Buenos Aires o La Plata, esta crisis tiene que ver con varias cuestiones superpuestas que ante todo refieren al número, con la emergencia de la cantidad, pero también de la heterogeneidad en lo que se superponen lo externo: proliferación de instituciones competitivas, la creación de un mercado en consecuencia de docentes, alumnos e instituciones que compiten por el prestigio y la jerarquía, la modificación de la relación la alta cultura-cultura popular, la heterogeneidad del alumnado vinculado a la masificación, las crecientes expectativas del conjunto de los actores societales y estatales respecto de la universidad.

De tal manera que una universidad sobredemandada, y esto vale también para los estudios de posgrado en el continente, que aún se representa a sí misma como una institución y no como una organización entre multiplicidad de nuevos modos y espacios intelectuales de formación e investigación. En lo interno esta universidad sobredemandada presenta tensiones difíciles de resolver que se hacen presentes en los aspectos pedagógicos, de socialización, de la localización de la investigación, de la relación grado-posgrado, de la relación entre teoría y práctica, entre estudios generales y especializados, entre investigación pura y aplicada y su localización en los planes y niveles, entre bordes disciplinarios vinculada a los nuevos contextos de aplicación del conocimiento; así como el surgimiento de nuevos establecimientos de producción de conocimiento y formación: las corporaciones, la educación a distancia, la implantación de sedes extranjeras, la emigración de estudiantes y científicos, etcétera.

La referida crisis es también de la institución y de la gestión y conducción, que hoy es más una gestión ligada a la dilución y gestión de conflictos que a una gestión capaz de orientar y dirigir el futuro. La crisis de hegemonía es de centralidad, se expresa también como crisis de legitimidad; es decir, de consenso societal y confianza, todo lo cual incide en lo institucional mencionado anteriormente.

La universidad latinoamericana más que amenazada, esta tensionada entre las demandas estatales, las de la sociedad y el mercado y los crecientes retos de la globalización apenas logran construir un perfil que le permita crear una nueva legitimidad. Como señalamos al principio, se hace necesario cada vez más recuperar la autonomía para replantear el compromiso desde una perspectiva crítica y utópica en la que la responsabilidad social y la producción de conocimiento 
ocupen un lugar central como intelectual colectivo, la sociedad de riesgo necesita de un dispositivo creador de nuevos valores y sentidos.

Para conocer y asumir este desafío se hace necesario comenzar a construir una profunda auto reflexividad, una reflexión sobre su propio ser, su estar ahí. Esta reflexividad le permitirá alejarse de la mera reproducción del presente, del patrón tradicional de universidad que hemos heredado.

Ese distanciamiento e implicación con el entorno, el dilema de los horizontes o fronteras, contemporaneidad con el futuro como fundamento de la universidad, debe superarse desde distintas

opciones. Sin duda, sólo la contemporaneidad con el futuro le permitirá a la universidad construirse como espacio público y crítico y como dispositivo auto reflexivo y autónomo de la sociedad. Este ha sido el mandato histórico que al decir de Ricoeur se ha encomendado a la universidad por la humanidad en algún momento de su larga historia. Para esto debemos cada vez más trabajar sobre nosotros mismos, "no solo domesticar lo extraño sino exorcizar lo doméstico", "objetivar al sujeto objetivante" pretensión de toda universidad que se precie como tal, construir una vigilancia epistemológica sobre su propio acontecer como quería Pierre Bourdieu (2005). En fin aventurarse a crear una nueva universidad argentina con rasgos latinoamericanos, aunque suene por demás ingenuo, que es una forma asimismo de construir la República en un futuro de integración con países de similares niveles de desarrollo de la región.

\section{Aterrizando y desanudándonos con los diversos ejes analíticos: escenarios deseables y posibles en prospectiva universitaria en el 2030 como tendencias}

Este apartado se apoya en varios ejercicios realizados con estudiosos de la temática sobre los futuros de la Universidad hacia el 2030 a lo largo de 2005 y el primer semestre de 2009 en la Universidad Autónoma Chapingo (UACh), en la Universidad Autónoma del Estado de México y en la Universidad Autónoma del Estado de Morelos. En estas dos últimas se trabajó especialmente con las facultades de ciencias agropecuarias. En 2008 se participó con un proyecto de la Universidad Autónoma Metropolitana (UAM) y la UNAM en el Centro de Investigaciones Interdisciplinarias en Humanidades de la misma UNAM. Se incluye también una reseña de nuestra participación (2006) en una discusión de expertos en la prospectiva de la educación superior mexicana, con el propósito de indagar escenarios hacia el 2030, organizado por el Conacyt de México. Desde luego se consideran las reflexiones al respecto que se señalan en algunos de los apartados anteriores de esta obra. Lo que a continuación se expone, son distintos escenarios deseables y posibles en el contexto de la época del informacionalismo (De Souza, Victorino, 2010), en los cuales los principales sujetos sociales y actores universitarios se organizan para alcanzar y concretar acciones pertinentes que la universidad latinoamericana requiere en el contexto global y nacional, con miras al año 2030. Estos escenarios pensados y ordenados como alternativas institucionales se organizan en función de seis 
variables o aspectos esenciales que conforman los ejes analíticos que desde el inicio de este ensayo y en algunos de los posteriores apartados de esta obra, se han señalado ${ }^{1}$.

\section{Ver para creer: las relaciones de producción}

$>$ La población latinoamericana alcanzará entre 15 y $20 \%$ de crecimiento poblacional a partir de 2010 hacia 2030. Un poco más de la tercera parte se ubicará entre la población económicamente activa (PEA).

> Habrá una economía con más rasgos de procesos de producción inmaterial, con énfasis en la producción de alimentos sin la necesidad de contar con la tierra, ni con los campesinos como elementos esenciales.

$>$ Se dará curso a una producción de alimentos altamente mediados por las ciencias bajo la investigación transdisciplinaria y en entornos más virtuales.

$>$ Si bien no desaparecen los campesinos, la función social del trabajo agropecuario transita de una práctica agrícola tradicional de rol fijo hacia una de multifuncionalidad agropecuariaecológica-rural y turística como expresión de roles flexibles del sector agropecuario.

$>$ Pese a ello, habrá un equilibrio entre el crecimiento de la macroeconomía y la microeconomía. El actual modelo económico de rasgos neoliberales, después de más de 30 años de incrementar pobreza, y pobreza extrema, ha tocado fondo. Aun con el regreso de gobiernos de derecha a los Estados nacionales de América Latina y a las presidencias de las repúblicas, el modelo neoliberal debe reorientarse y considerar recuperar el mercado interno, así como apoyar a los sectores rurales y urbanos más necesitados. El Estado debe invertir en aquellos rubros de infraestructura y de desarrollo donde la empresa privada ha mostrado ineficiencia, o incluso corrupción. En fin, se implementará una economía con dosis de desarrollo social y humano.

$>$ La población que más demanda educación (de 4 a 25 años) se reducirá en términos absolutos, en cambio la población que más demanda empleo, fluctuará entre de 25 y 64 años, casi se duplicará su cantidad en la región.

$>$ El perfil de la PEA de México se aproximará más a la de los países miembros de la OCDE, y se diferenciará un poco de la media frente a varios países latinoamericanos. Dicho perfil se acompañará de una expansión de la educación principalmente de la media superior y superior, incluyendo el posgrado.

$>$ El carácter nacional de algunas universidades públicas de la región, se apoya en las funciones sustantivas, así como en los dispositivos académicos que despliega articuladamente con

\footnotetext{
${ }^{1}$ Algunas de estas aseveraciones son retomadas por las reuniones que sostuvimos durante el Seminario de Educación Agrícola y Vinculación Universitaria, del 19 de febrero al 15 de junio de 2009. En especial se consideran algunos planteamientos de la sesión del 14 de mayo de 2009 con profesores e investigadores de la Facultad de Ciencias Agropecuarias de la Universidad Autónoma del Estado de Morelos, celebrado en Cuernavaca, Morelos, México.
} 
el compromiso académico y social de contribuir a la revaloración, tanto de la educación como la investigación, el mundo urbano y rural, la agricultura y los sujetos de los distintos campos productivos como factores cruciales del desarrollo nacional de los distintos países.

\section{Divide y reinarás: relaciones de poder}

$>$ Se avanzará hacia la descentralización educativa, económica, política y social. Se transitará de la desconcentración a la descentralización. Los Ministerio de Educación Pública mantienen nuevas relaciones tanto con las otras instancias gubernamentales como con las organizaciones laborales adscritas a las universidades públicas.

$>$ Todavía sin acuerdo migratorio, pero el gobierno mexicano tendrá más influencia en el respaldo laboral para los migrantes de los consulados nacionales en el extranjero, especialmente en EU. El flujo migratorio de México a EU bailará al ritmo de las crisis coyunturales y estructurales, lo que implica que dependiendo del ritmo de crecimiento ocuparán más o menos mexicanos y latinoamericanos en trabajos temporales. México sigue siendo el principal puente de enganche para nuestros hermanos latinoamericanos, especialmente centroamericanos.

$>$ América Latina con sus distintas orientaciones ideológicas en función de los bloques descritos en este ensayo, sigue con la idea de su integración como continente próspero y revolucionario. México marchará de una democracia formal a una democracia participativa: habrá muchos pequeños partidos regionales y estatales-locales, y contadas coaliciones que participarán preferentemente en las elecciones presidenciales nacionales.

$>$ Se incrementará la diferenciación de las universidades a partir del uso de las TIC en sus funciones sustantivas. Las universidades federales, nacionales, públicas y autónomas con más población y más presupuesto serán las cabezas del desarrollo de la educación superior a distancia (ESaD). En México, por ejemplo: la UNAM, la Universidad Autónoma Metropolitana (UAM) y el Instituto Politécnico Nacional (IPN) como las principales IES públicas, llevarán la delantera en el desarrollo de programas y proyectos de ESaD como nuevo paradigma educativo con filosofía propia.

$>$ No se podrá evitar la venta de servicios educativos como simple negocio de las grandes instituciones educativas privadas como el Instituto Tecnológico de Estudios Superiores de Monterrey (ITESM), por cierto con mucho impacto en las universidades públicas de la región, el cual desde hace 20 años ya promueve la universidad virtual y ofrece diplomados a los gobiernos federales y estatales en el centro y norte del país, e incluso en varios países de América Latina.

$>$ Las universidades públicas de la región, especialmente en sus estudios de posgrados, asientan su carácter crítico en la autoridad ética e intelectual resultante del desarrollo de un modelo académico en el que la docencia, la investigación, el servicio y la difusión de la 
cultura convergen en la generación de explicaciones, previsiones y respuestas integrales, académicamente consistentes y socialmente relevantes para la sociedad en su conjunto.

$>$ El ejercicio responsable de la autonomía universitaria se refleja plenamente en la consistencia de su sistema democrático de gobierno, en la relevancia que asumen los asuntos académicos en la toma de decisiones colegiadas, en la congruencia del funcionamiento académico administrativo, así como en la participación de la comunidad universitaria a través de los distintos órganos colegiados en los que está representada.

\section{Como lo vi, lo cuento, o el mitote de la experiencia humana y movimientos sociales}

$>$ Los movimientos sociales protagonizados por organizaciones estudiantiles, campesinas, magisteriales y sectores de la clase media, fortalecerán sus formas de lucha por distintos medios. Sin duda uno de los más recurrentes será el uso de las TIC mediante las redes sociales y la red Internet sobre todo en la solidaridad internacional de sus organizaciones hermanas en distintos países del mundo. En prospectiva el Foro Social Mundial no desaparecerá, sino al contrario se revitalizará en busca de que "otra globalización es posible". A nivel nacional en el caso mexicano, el pasado mes de octubre de 2009 la lucha de los twiteros y toda la comunidad de la red de Internet impidieron que los diputados y el gobierno federal grabaran impuesto a los usuarios de la Red de Internet de México. Esto no es una muestra exitosa de la revolución y el uso de las TIC en la lucha social.

$>$ Se fortalecerá el respeto a los derechos humanos y sociales en general, porque entre otras medidas, existe una vigilancia de organismos internacionales de diferentes ideologías para su aplicación y transparencia y obviamente crece la organización y lucha de estos denominados grupos minoritarios.

$>$ Especialmente los llamados derechos de los grupos autodenominados minoritarios tomarán fuerza social por la legitimidad de sus acciones y demandas en contra de los problemas de inseguridad, respetos a los derechos humanos, corrupción y justicia social.

$>$ Las formas de convivencia que hasta hace 10 años eran inconcebibles e inaceptables como el matrimonio entre personas del mismo sexo, entre otros, será cada vez más reconocido mediante la vía legal y legítima en el contexto del respeto de los derechos humanos.

\section{Sin quitar el dedo de renglón, los cambios culturales}

$>$ El desarrollo, preservación y difusión de la cultura sintetizan de manera primordial la pluralidad de funciones que cumple la universidad en latinoamérica, creando los principales productos de su quehacer académico mediante una comunicación dialógica con las sociedades rurales, las comunidades epistémicas o científicas, intelectuales, la opinión pública en general y la propia comunidad universitaria que aumenta los beneficios de la cultura, la 
participación crítica de la sociedad en su desarrollo, así como la presencia y proyección de los fines universitarios.

$>$ Las tecnologías de la información y comunicación forman parte de una realidad, tanto para nuestra vida cotidiana como para el cumplimiento de las funciones sustantivas y adjetivas de la universidad, puesto que han ensanchado las capacidades físicas y mentales, así como las posibilidades de desarrollo social. Los continuos avances científicos y tecnológicos en el contexto de la globalización económica y cultural, contribuyen a la rápida obsolescencia de los conocimientos y a la emergencia de nuevos valores, provocando continuas transformaciones en nuestras estructuras económicas, sociales y culturales y repercutiendo en casi todos los aspectos de nuestra vida: el acceso al mercado de trabajo, la sanidad, la gestión burocrática, la gestión económica, el diseño industrial y artístico, el ocio, la comunicación, la información, la manera de percibir la realidad y de pensar, la organización de las empresas e instituciones, sus métodos y actividades, la forma de comunicación interpersonal, la calidad de vida y la educación.

$>$ Las TIC se utilizarán más en investigación inter y transdisciplinaria, cine, televisión (TV) y seguramente en los libros y, aunque no totalmente, en los programas académicos de las universidades. Aunque no desaparecerá la publicación de libros impresos, serán más dinámicos y eficaces las revistas y libros electrónicos.

$>$ La generación punto $\mathrm{X}$ y punto Y estarán ascendiendo a la toma de decisiones en distintos rubros de la cultura científica. Esto será un factor determinante para la incorporación de las TIC en distintos órdenes sociales y culturales.

$>$ Las TIC se usarán más en el trámite administrativo académico y laboral, aunque eso no es necesariamente $\mathrm{ESaD}$. Los sistemas educativos convivirán entre la educación convencional y la educación virtual.

$>$ Las TIC, traducidas como herramientas para el aprendizaje y la enseñanza, han incorporado la educación a distancia o virtual a los planes y programas de estudios en los distintos niveles educativos (bachillerato, licenciatura y posgrado) de la universidad latinoamericana. Con el apoyo de esta modalidad educativa se ha logrado incrementar la matrícula universitaria al menos en un $100 \%$ comparado con la del 2009 , y su proceso de desconcentración ha configurado a la universidad latinoamericana y sus posgrados como una universidad con grandes centros de aprendizaje y de auto acceso en todas las sedes, preferentemente en sus Unidades y Campus propios de las universidades de los países de la región.

$>$ Seguirán promoviendo desde los grupos étnicos de los distintos países una tendencia hacia la no discriminación de los sectores marginados, hacia el fortalecimiento y demandas de apoyo gubernamental de los pueblos originarios, especialmente por el respeto de los pueblos indígenas y sus propias formas de estructura agraria y organización autónomas como símbolo de identidad nacional en el contexto de los países pluriculturales. 


\section{A ciencia y paciencia, la educación superior y de posgrado}

$>$ Los niveles de calidad alcanzados por la universidad latinoamericana están en correlación directa con los atributos que definen el funcionamiento holístico de sus modelos académicos, que integra a la investigación, la docencia, el servicio y la difusión de la cultura. De igual modo, se asientan en la intensa participación que despliega el personal académico en el desarrollo integral de las actividades sustantivas universitarias, así como en la existencia y operación de un sistema institucional de gestión de la calidad.

$>$ La docencia se cimienta en un modelo de formación que, reconociendo las funciones básicas de los tres niveles educativos que imparte la universidad, se orienta a la construcción del aprendizaje desde una enfoque integral con los aportes de la investigación, el servicio y la difusión de la cultura, para responder a las continuas demandas del desarrollo del conocimiento y la formación universitaria. Bien se puede decir que esta intencionalidad ya se viene desarrollando por algunos sectores académicos desde 1990.

$>$ El servicio universitario se constituye en la actividad académica articuladora por excelencia de los aportes de las demás funciones sustantivas y con perspectivas propias, potencia su influencia directa y socialmente comprometida con el desarrollo integral de los sectores más desprotegidos del medio rural. Este escenario reconoce intentos académicos significativos con experiencias desde la década de los setenta del siglo XX.

$>$ La universidad, bajo el principio de pluralidad, sostiene una amplia y diversa red de vínculos con la sociedad mexicana y con comunidades académicas, científicas tanto nacionales como extranjeras que abren nuevas oportunidades de desarrollo al continuo enriquecimiento de sus funciones sustantivas. La vinculación universitaria será reconocida como una más -posiblemente la $5^{\text {a }}$ función- de las funciones sustantivas de las universidades y esto será motivo de modificar sus objetivos y funciones fundamentales de las IEAS.

$>$ Se ampliará la cobertura en educación superior en todos los países, especialmente en México crece de $17 \%$ a $35 \%$ y sigue la disputa entre la educación superior pública y la privada o particular.

$>$ El sistema educativo superior en la seguirá feminizándose en las distintas universidades del continente. En México pasará de 30\% a 48\%.

$>$ En el mejor de los casos, en varios posgrados de las universidades de A.L. apenas 10\% de la matrícula en el nivel de educación superior posgradual formal gozará la modalidad de ESaD. Es decir, las TIC seguirán usándose marginalmente en esta modalidad educativa.

$>$ En las universidades públicas estatales y autónomas ya no habrá construcción de grandes edificios para ampliar su cobertura. Estarán descentralizadas, existirán pequeñas universidades municipales y fungirán como centros de acceso donde no se concentrarán grandes grupos de estudiantes y profesores como lo hacían en las formas tradicionales. Los profesores serán sustituidos por tutores o facilitadores. 
$>$ Se transitará entonces del proceso de enseñanza-aprendizaje al de aprendizaje y enseñanza semipresencial y virtual.

$>$ Las universidades públicas de la región sigue el proceso de descentralización. Estos serán campus regionales con carreras de fortalecimiento nacional, y sobre todo con unidades locales, pero de impacto educativo regional en A.L.

\section{A ciencia cierta, la generación y producción de materia gris}

$>$ La generación, producción y aplicación de nuevos conocimientos se constituye en el eje dinamizador del modelo académico, ya que la investigación aporta de manera sistemática innovaciones académicas y científicas que contribuyen a la permanente transformación de las perspectivas y prácticas académicas, prácticas docentes, al fortalecimiento de los cuerpos académicos, redes temáticas, centros de investigación, al desarrollo de los campos científicos y tecnológicos estratégicos para el país, y a la posibilidad de generar soluciones, alternativas, así como las necesarias previsiones que exige el desarrollo rural integral y sustentable.

$>$ La formación de los nuevos docentes e investigadores versará en torno al manejo de las guías didácticas, el diseño instruccional, la elaboración de unidades pedagógicas (antologías virtuales), redes de bibliotecas digitalizadas, y la investigación se situará en redes sociales de investigación y generación de conocimientos.

$>$ La investigación social y política ya no tomará como objeto de estudio o de indagación al Estado Nacional, ni sólo a las políticas gubernamentales sino que buscará más la realización de investigación comparada de las acciones gubernamentales, públicas y privadas del Estado-red global.

$>$ Se fortalecerán nuevas áreas de generación y producción de conocimientos que hoy se presentan como las emergentes: investigación en la biodiversidad, en bioenergéticos, el impacto de las TIC y los derechos humanos, la producción de alimentos en la agricultura inmaterial, y marginalmente en la agricultura tradicional, biotecnología y producción de alimentos, energías renovables, estudios sobre el calentamiento global, las opciones alternativas frente al cambio climático, la opción de un mejor aprovechamiento social del agua, y alternativas en general, entre otros temas interesantes.

$>$ Las carreras y los estudios de posgrados de las universidades del futuro en la modalidad de ESaD buscarán de inmediato su acreditación de Calidad, nacional primero e internacional después, que serán evaluados con indicadores de impactos en el marco de referencia de organismos nacionales expertos en evaluación, acreditación y certificación.

$>$ La organización de las comunidades epistémicas o científicas se fortalecerán con las redes sociales de generación y producción de conocimientos. Se avanzará en el modo 2 de la investigación en contexto de aplicación e implicaciones sociales propuesta por la UNESCO 
desde 2002. Así como en las propuestas de generación y construcción de conocimientos en contextos de aplicación y sus implicaciones contenidas en esta obra. Las redes sociales de investigación se estructurarán en redes temáticas constituidas por diversos enfoques multi, inter y transdisciplinarios interinstitucionales tanto nacionales como con universidades y centros de investigación de otros países.

$>$ La investigación educativa como una de las áreas prioritarias de la investigación en la universidades, es y seguirá siendo esencial para el autoconocimiento de las universidades y sus alternativas de cambios, para conocer el impacto en las trayectorias escolares, en el seguimiento de egresados, en las repercusiones de las políticas de financiamiento, calidad, evaluación, acreditación, de la modalidad de educación virtual o a distancia y de las nuevas carreras vinculadas a la cuestión ambiental y al desarrollo sustentable.

Aunque no se precisan las circunstancias del por qué se tomó como referencia el lapso de 20102030 para la construcción de los escenarios, reiteramos que es un periodo de largo plazo en el tipo de planeación prospectiva y porque los grandes escenarios posibles y deseables desde la filosofía y misión de las universidades no deben perder la brújula del horizonte.

\section{Reflexiones finales}

Las principales dimensiones analíticas, entendidas como germen de cambios, en el estudio sobre la universidad latinoamericana se mueven constantemente, siguiendo la disputa entre dos grandes tendencias ideológicas; en lo universal y en lo singular, que se concretan en la concepción de la educación y de la universidad: como bien social público o como una educación para la rentabilidad y el negocio. Nosotros nos movemos en la primera apreciación.

En cuanto a las nuevas fuerzas del cambio, son muchos los obstáculos por superar para lograr una verdadera incorporación de innovaciones y cambios en la vida universitaria, como entre otras la educación superior a distancia (ESaD), verbigracia. Es muy difícil que cada una de las universidades públicas estatales, especialmente el posgrado latinoamericano se pongan a la vanguardia en esta modalidad educativa. Lo más probable, ante la cada vez difícil consecución de financiamientos para programas nuevos, es que se forme una Universidad Nacional a Distancia ex profeso, (con apoyo de universidades públicas autónomas de mayor experiencia en la materia) en el seno de los ministerios de educación de los países que conforman la región, y ofrezca servicios de formación y capacitación a las universidades estatales públicas autónomas. Acopiar recursos para concentrarlos en una nueva universidad pública nacional es más viable que una diversidad de apoyos para programas como éstos, que en algunos casos, las autoridades universitarias lo aprovechan para cubrir el proyecto que más le interese a la administración en turno.

Queda por lo menos entendido que en las diversas universidades de la región el encuentro de las políticas sociales más impactantes en el campo de la educación asumen dos tendencias: 
aquellas que se recepcionan de manera pasiva e introducen cambios casi sin la participación de los profesores e investigadores; y aquellas otras en donde los propios docentes e investigadores diseñan y participan activamente, con dosis de resistencia, que tratan de mejorar la calidad de las universidades en el continente. Todo este conjunto de ideas y acciones que han trasminado los distintos tipos de reformas universitarias: estructurales y coyunturales, han venido a poner en crisis el mismo concepto de universidad. Estos pensamientos de futuro en los hechos cambian la "idea de universidad" que se proclamó en el industrialismo o en la modernidad, tanto en el modelo francés (Napoleónico), como en el modelo alemán, al entenderse a la universidad como "la más clara conciencia espiritual de la época". No obstante, autores brasileños anticipaban esta visión de futuro como Ribeiro (1971) y actualmente Boaventura Sousa (1994), al plantear sabiamente cambiar "la idea de universidad" por la de "universidad de las ideas" e incluso este último, al analizar las limitaciones del conceptos de universidad tradicional, nos habla de una "pluridiversidad" abierta al tiempo y a los grandes y complejos cambios sociales, como el avance científico-tecnológico, las nuevas formas de generación y producción de conocimientos, entre otros.

Si se prefiere, lo expuesto en este ensayo puede leerse como un sueño o como una utopía social. Decimos que puede ser, pero dejando claro que es un sueño bajo el "principio esperanza" y una utopía social pero no en la vieja concepción marxista del término, de lo que no puede ser, sino una utopía social como "el bien de lo posible", de lo que podemos realizar. Por lo tanto las bases de las propuestas están consideradas en este capítulo, que puede fungir, en el mejor de los casos, como una guía para la acción universitaria del desarrollo en los próximos veinte años.

\section{Bibliografía}

Banco Mundial (1998/1999). World Development Report: Knowledge for Development, pp.1-14. Bourdieu, P. (2005).El oficio de sociólogo. México: Siglo XXI.

Busch, L.; Wiliam B. Lacy; Jaffrey Burkhardt y Laura R. Lacy (1991). Plants, Pawer, and Profitts: social,economic, and ethical consequences of the new biotechnologies. Cambridge, Massachusetts: Basil Blackwell.

Burton, C. (1998). El sistema de educación superior. México: Siglo XXI. (1999). Las universidades modernas: espacios de investigación y docencia. México: Siglo XXI.

Brunner, J. J. (2007). "Universidad y Sociedad en América Latina”, Universidad veracruzana, México, (versión electrónica, HYPERLINK. Recuperado de http://www.universidadveracuruzana.com)

Castells, M. (1998). End of Millenimum. USA: Rowmans \& Littlefield Publishers. (2000). La era de la información. Educador, Barcelona: Paidós.

Collis, B. (1999). “Tecnología de la información en la educación”. Universidad de Barcelona, España. Recuperado de www.voc.edu/rusc/esp(collis.html.6k). 
Liberio Victorino Ramírez, Rocío Ángeles Atriano Mendieta y Héctor Rueda Hernández

CONACYT (2006). Padrón Nacional de Posgrado de Calidad. México.

De Alba, A. (comp.). (1995). El curriuculum universitario de cara al nuevo milenio. México: SEMARNAP-UNAM.

(2009). La crisis estructural generalizada. En: Orozco, Berta (coord.). Configuraciones y experiencias curriculares. México: IISUE-Plaza y Valdez, pp. 13-45.

De Souza S., J. (1988). The contradictions of the Biorevolution for the Develment of Agriculture in the Third Wold: biotecnology and capitalist entrests. Agriculture and Human Values, Summer, pp. 61-70.

- (1996). From Medicinal Plants to Natural Pharmaceuticals: the markenting of nature. En: Pan American Health Organization Biodiversity, Biotecnology, and sustainable Development in Health and Agriculture: Emerging connetions. Washignton: PAHO, pp. 109-129.

— et al. 2004. La cuestión institucional. Costa Rica: IFPRI-RNPDIAL.

— J. Santamaría; M. A. Mato y A. León (2001). La dimensión de estrategia en la construcción de la sostenibilidad institucional. Serie Innovación para la Sostenibilidad Institucional. San José, Costa Rica: Proyecto ISNAR "Nuevo Paradigma”.

ECOESAD (2008). La oferta educativa en licenciatura en el Espacio Común de Educación Superior en México. Recuperado de www.RIISAED-ECOESAD.COM

Escurra, A. M. (2008) ¿Qué es el neoliberalismo?. Evolución y límite de un modelo excluyente. Lugar editorial IDEAS, Argentina.

Follari, R. (1999) La interdisciplina en la educación ambiental. Tópicos en educación ambiental, Volumen no. 2, SEMARNAP-UNAM, México.

Freire, P. (2003). Hacia una Pedagogía de la pregunta: conversaciones con Antonio Faúndez. Buenos Aires: Ediciones La Aurora.

García G., C. (2000). Balance de la década de los 90 y reflexiones sobre las nuevas fuerzas de cambio en la educación superior. Caracas: Universidad Central de Venezuela.

Goonatilake, S. (1984). Abortes Discoveries: Science and Creativity in the Third World. Londres: Zed Press.

González C., P. (2002). La universidad del siglo XXI. México: Siglo XXI, Editores.

Joy, B. (2000). "Why the future Doesn't Need Us". Recuperado de http://www.wirednerws.com/ wired/8.04/joy.html.

Held, D. (1980). Aportaciones a la Red Nuevo Paradigma. En: La innovación de la innovación institucional. EUA: COSUDE-IFPRI-RED NUEVO PARADIGMA, Universidad de Carolina del Norte, pp. 57-67.

- y A. McGrew (2000).The Global Transfornations Reader: on intrucction to the globalization debate. Cambridge: Polity Press.

Hill, C. (1969). Reformation and Industrial Revolution. Londres: Weidenfeld \& Nicolson.

Hobsbawm, E. (1981). Looking Forward: history and the future. Londres: New Left Review. 
Politicas universitarias y neoliberalismo. Resultados y tendencias en la universidad latinoamericana

Kent S., R. (2005). La política de la esperanza y la desilusión de la educación superior en México. Revista de la Educación Superior, vol. XXXIV (2), abril-junio, México: ANUIES, pp. 63-79. Krotsch, P. (2009). "Conferencia en la Universidad Nacional de Córdoba". Argentina, 4 de junio de 2006 en Boletín ALAS, núm.6, agosto-septiembre de 2009.

Langlois, R. N. (1997). Capacidad y gobernanza: el renacimiento de la producción en la teoría de la organización económica. Vol. 52, Londres: Blakwel Publishing.

Labbe, C. G. 2003 "La Educación Virtual en Chile: Historia, estado del arte y proyecciones". En: Seminario Internacional sobre Universidades Virtuales en América Latina y el Caribe. Quito, Ecuador: IESALC, 13-14 de febrero.

Miklos, T. y Tello, M. E. (1996). Planeación prospectiva. Una estrategia para el diseño del futuro. México: Fundación Barros Sierra, A.C., Editorial Limusa.

Morín, E. (1999). Los siete saberes necesarios para la educación del futuro. Paris: UNESCO.

Osorio, F.; Marcelo, A.; González, S. y Aguado, E. (coords.) (2008). La nueva Teoría Social en Hispanoamérica. Introducción a la Teoría de Sistemas Constructivistas. México: Colección Pensamiento Universitario, núm. 11, CEU-UAEM.

Rifkin, J. (2000). La era del acceso. USA. Putnam Publishing Group.

Rouseau, J. J. (1985). El Contrato Social. Barcelona: Editores Mexicanos Unidos.

Santamaría, M. y Sánchez-Elvira, A. 2004. Formación permanente y enseñanza en-línea. Curso de Experto en Administración de la Educación. Madrid: Módulo I. UNED.

Sartori, G. (2007). Homo Videns. La sociedad teledirigida. México: Punto de Lectura.

Sen, A. (1997). Desigualdad y desempleo en la Europa contemporánea. Revista Internacional del Trabajo. 116 (2), pp.167-187.

Silvio, J. (2003). La virtualización de la universidad: ¿cómo podemos transformar la educación superior con la tecnología? Caracas: Ediciones IESALC-UNESCO.

Stevenhaguen, R. Conferencia "Los Pueblos Indígenas y los Derechos Humanos". En: Seminario La Sociología Rural en el Mundo de hoy. DEIS Sociología Rural, UACh, del 25 al 29 de enero, Texcoco, Estado de México.

Time Almanac. (1998). Gane Piracy: some ecologists are sounding dark wranings of a coming gane war between industrialized and emerging nations, november 30, pp. 20-25.

Universidad Autónoma Chapingo (2001). Programa de Desarrollo Universitario, 2001-2025. México.

- (2009). Plan de Desarrollo Institucional, 2009-2025. México.

Victorino R., L. (2008). Nuevo paradigma educativo en educación superior a distancia en el porvenir. Revista Geografía Agrícola, núm. 40, UACh, México, pp. 35-50.

- (1998). Los investigadores sociales ante el cambio. Problemas y expectativas. México: UACh. (2006). Procesos de evaluación de la Universidad en Hispanoamérica. La experiencia de los 90. México: UACh. 
(2008). Puntos críticos en la agenda de la educación. Las universidades públicas y autónomas de la región centro sur de México. México: UAEM.

Wallerstein, I. (1999). The End of the World as We Know It. USA: Minneapolis, University of Minnesota Press.

Recibido: 15/05/14

Dictaminado: 26/10/14

Corregido: 06/11/14

Aceptado: 07/11/14 